\title{
Effect of Low-load resistance training with different degrees of blood flow restriction in patients with knee osteoarthritis: study protocol for a randomized trial
}

\section{Haonan Wang}

Beijing Sport University

Lin Cheng

Beijing Sport University

\section{Yan Chen}

Beijing Sport University

\section{Shen-Tao Wang}

Beijing Sport University

De-Xin Hu

Beijing Sport University

\section{Li-Na Wang}

Beijing Sport University

Guo-Xin Ni ( $\square$ niguoxin@bsu.edu.cn )

Beijing Sport University

\section{Study protocol}

Keywords: Knee osteoarthritis, Blood flow restriction, Vascular occlusion, Resistance training,

Rehabilitation

Posted Date: May 10th, 2021

DOl: https://doi.org/10.21203/rs.3.rs-467579/v1

License: (c) (1) This work is licensed under a Creative Commons Attribution 4.0 International License. Read Full License

Version of Record: A version of this preprint was published at Trials on January 3rd, 2022. See the published version at https://doi.org/10.1186/s13063-021-05946-7. 


\section{Abstract}

Background: Knee osteoarthritis (KOA) is a common degenerative disease that causes pain, functional impairment, and a reduced quality of life. Resistance training is considered an effective approach to reduce the risk factor of muscle weakness in patients with KOA. Blood flow restriction (BFR) with low-load resistance training have better clinical outcomes than low-load resistance training alone. However, the effective degree of BFR with low-load resistance training has not been determined. The purpose of this study will be to evaluate the effectiveness of different degrees of BFR with low-load resistance training in patients with KOA on pain, self-reported function, physical function performance, muscle strength, muscle mass, and quality of life.

Methods: This is a study protocol for a single-blind, randomized, controlled trial. One hundred individuals will be indiscriminately assigned into the following groups: two training groups with a BFR at $40 \%$ and $80 \%$ limb occlusion pressure (LOP), a training group without BFR, and a health education group. The three intervention groups will perform strength training for the quadriceps muscles twice a week for 12 weeks. The health education group will attend sessions once a week for 12 weeks. The primary outcomes will include pain, self-reported function, and adverse events. The secondary outcomes will include physical function performance, muscle strength of the knee extensors, muscle mass of quadriceps, and quality of life. The intention-to-treat analysis will be conducted for individuals who withdraw during the trial.

Discussion: Previous studies have shown that BFR with low-load resistance training is more effective than low-load resistance training alone; however, a high degree of BFR may cause discomfort during training. If a 40\% LOP for BFR will produce similar clinical outcomes as an $80 \%$ LOP for BFR, resistance training with a low degree of BFR can be chosen for those patients with KOA who are intolerant to a high degree of BFR.

Trial registration: Chinese Clinical Trial Registry, ChinCTR2000037859. Registered on 2 September 2020.

\section{Background}

Knee osteoarthritis (KOA) is one of the most common chronic musculoskeletal diseases. It can cause the subchondral bone, cartilage, synovium, and menisci [1], which may result in pain, stiffness, impaired function of daily activities, and a decline in the quality of life (QoL). According to an epidemiological survey, it is estimated that the prevalence of symptomatic radiographic KOA among older people is $12.1 \%$ in the US [2] and $8.1 \%$ in China [3], and these figures are showing trends for increasing. Due to its high prevalence, KOA may cause a huge economic burden on health services, such as conventional interventions and joint replacements [4]. Therefore, primary and secondary prevention programs are necessary to minimize social and personal costs.

Multiple risk factors may contribute to the development of KOA, such as age, sex, obesity, and various joint factors; unfortunately, the link between these factors and KOA remains unclear [5-7]. However, muscle weakness, especially in the quadriceps, is considered both a vital and modifiable risk factor for 
KOA [8]. Previous studies have shown that muscle weakness is strongly associated with the incidence [8] and progression [9] of KOA, as well as the physical function and knee pain of individuals [10]. Moreover, muscle mass in the lower limb is independently influenced by the presence of KOA [11] and related to the severity of symptoms in patients [12].

The main approach to improve muscle strength and mass is resistance training (RT). RT can decrease the risk of KOA by increasing muscle strength and muscular hypertrophy [13]. Based on the recommendations of the American College of Sports Medicine, a resistance load of $60-80 \%$ of an individual's one-repetition maximum (1RM) is necessary to achieve muscle hypertrophy and improvements in muscular strength [14]. However, high load resistance training (HLRT) can cause aggravating pain and joint deterioration in patients [15], which result in a decreased compliance for therapeutic exercises and a slower rehabilitation process.

Currently, blood flow restriction (BFR) training has drawn the attention of clinicians and physiotherapists in the field of musculoskeletal rehabilitation, and it is believed to be an alternative approach for HLRT. In short, a pneumatized cuff, or tourniquet, is used to block partial arterial blood flow to the limb and this is combined with 20-30\% 1RM low-load (LLRT). With an ischemic state in the limb, BFR training will produce a stronger physiological metabolic stress that includes growth hormone $[16,17]$ and an increased recruitment of type II muscle fibers [18]. Due to this, even though the load is relatively low during the BFR training, it can still produce increases in muscle strength and mass, similar to the results seen with HLRT. Importantly, BFR training requires a lower external load, which is beneficial for decreasing the joint loading and tolerance of RT.

Previous studies have found that BFR in combination with LLRT shows better improvements in muscle strength compared to LLRT alone in patients with a risk of $\operatorname{KOA}[19,20]$, while it has similar effectiveness as HLRT in increasing muscle strength, muscle mass, and function in patients with KOA [21, 22]. However, only one previous trial has used the percent of limb occlusion pressure (LOP) to identify the pressure for BFR training in patients with KOA [22], other studies have used the absolute air pressure of the cuff. It is worth noting that a variety of factors may contribute to the LOP, including systolic blood pressure, diastolic blood pressure, sex, limb circumference, limb length, and cuff width [23, 24]. Therefore, the application of LOP can produce a more precise and similar arterial occlusion for different individuals when compared with an absolute air pressure. It has been shown that BFR pressures with $40 \%$ are effective in an acute response of muscle [15], but the ability to achieve a chronic response with BFR remains unclear, especially for patients with KOA. Additionally, while a higher LOP promoted more pain during exercise with BFR training[25], it seems that a pressure lower than 70\% LOP may be more comfortable for individuals and increase the compliance of the intervention program.

There is still a lack of evidence concerning the lower LOP percent, which is more effective than LLRT alone, but presents less discomfort for the patients. Therefore, the aim of this study will be to investigate the efficacy of LLRT with different degrees of BFR in patients with KOA. Our measures will include pain, self-reported function, physical functional performance, muscular strength, and muscle mass. We will 
also observe any adverse events to verify the safety of BFR training during the study. As health education is also important for KOA, we will set a control group that will receive educational sessions and leaflets related to protecting their knees during daily activities.

Hypotheses:

1. The LLRT, LLRT with low BFR (40\% LOP), and LLRT with high BFR ( $80 \%$ LOP) will all be more effective than health education.

2. Both LLRT with $40 \%$ BFR and $80 \%$ BFR will be more effective than LLRT alone, but there will be no difference between the two levels of BFR.

\section{Methods}

\section{Study design}

This study will be a prospective, single-blind, randomized, controlled trial conducted in Beijing Chaoyang Hospital. The measurements of several outcomes will be made before and after the interventions and 24 weeks after the interventions begin (Fig. 1). This study was approved by the Ethics Committee of Beijing Sport University (Ethics Approved Number: $2020108 \mathrm{H}$ ) and registered at the Chinese Clinical Trial Registry (ChinCTR2000037859). We designed this study using the Standard Protocol Items: Recommendations for Interventional Trials (SPIRIT) statement [26] (Fig. 2) and the results will be reported in accordance with the CONsolidated Standards of Reporting Trials (CONSORT) guidelines [27].

\section{Participants}

In this study, we will recruit 100 patients, both men and women, with the diagnosis of unilateral or bilateral KOA. This group will then randomly be assigned to different interventions. The participants will be recruited using targeted strategies, including social media (WeChat), websites, newspapers, and other community approaches. If an interested patient meets the eligibility criteria, then the informed consent process will be performed. All potential participants will be informed that the study will last for 6 months and they have the right to withdraw at any time. If they are uncertain whether they will be able to participate for the entire 6-month period, we will provide regular healthcare for their KOA rather than include them into the formal study. Figure 1 demonstrates the flow chart for the trial, which includes participant recruitment, eligibility screening, baseline measurements, randomization and allocation, intervention, and outcome assessments. The enrollment of participants, the randomization procedure, and the performance of various measurements will be conducted by four independent physiotherapists. A manual will be developed to standardize the administration of the subjective questionnaires and the objective performance tests.

\section{Inclusion criteria}


The inclusion criteria will be as follows: (1) 45-75 years of age, (2) unilateral or bilateral KOA diagnosed according to the American College of Rheumatology clinical classification system [14, 28], (3) radiologic confirmation of KOA demonstrating Kellgren-Lawrence grade II or III [29], (4) average pain intensity of 40 or more on a 100-mm visual analogue scale (VAS) in the last week, and (5) adequate Mandarin language skills to complete the Western Ontario and McMaster Universities Osteoarthritis Index (WOMAC) and a written informed consent.

\section{Exclusion criteria}

The exclusion criteria will be as follows: (1) a history of knee surgery or scheduled surgery; (2) a history of any invasive procedure of the affected knee, including arthroscopy or intra-articular injection in the past 12 months; (3) a history of physical therapy/physiotherapy or a strengthening procedure of the affected knee in the past 6 months; (4) use of nonsteroidal anti-inflammatory drugs in the past 3 months; (5) any neurological, heart, or vascular disease, such as blood coagulation disorders; (6) abnormal blood pressure (resting systolic blood pressure [SBP] $>160$ or $<100 \mathrm{mmHg}$ or a diastolic blood pressure [DBP] $>$ $100 \mathrm{mmHg}$ ); or (7) other acute or chronic disorders or psychiatric conditions that will affect physical or cognitive functions.

\section{Withdrawal criteria and management}

KOA patients will be asked, or be allowed, to quit the study in the following cases: (1) participants have such a demand and (2) there is a severe adverse event during the study.

\section{Randomization and allocation concealment}

Eligible patients will be randomly assigned to one of the following groups: LL group, High BFR (80\% LOP) group, Low BFR (40\% LOP) group, and HE group, at a ratio of 1:1:1:1. The research staff will independently use SAS software (SAS Institute, Cary, NC, USA) to generate a randomization sequence and this staff will not participate in the intervention or statistical analyses of this study. The number sequence will then be placed into a sealed opaque sequentially numbered envelope prior to the study by another independent research assistant who will also not participate in other parts of this study. Once eligible patients with KOA finish the informed consent process, the demographic information and baseline measures will be recorded. Next, the research coordinator, who will not be involved in the measurement process, will have the authorization to open the envelopes in ascending order to determine a patient's intervention group assignment.

\section{Masking}

The measurement assessors will be blinded to the assignment group and work in the university remote to where the intervention will be performed. The statistician will be blinded to the group information of original data. Participants will be informed that all interventions have proven efficiency for their knee disorder, but it is still unknown which intervention is better. Moreover, they will be asked to not discuss their intervention and group assignment at any time during the study. Unmasking will only be allowed in the case of severe adverse events and will be reported as a part of the results of this study. 


\section{Intervention}

All participants of the intervention groups will complete 24 exercise sessions over 12 weeks with 2 sessions per week as described in Table 1. During the 12 weeks, participants may continue their previous activities without aggravating their knee symptoms. In an individual exercise session, each group will perform a warm-up exercise to prevent injuries before the formal exercise protocol. Moreover, the pain intensity will be monitored by a VAS during the exercise, with some pain being acceptable during the exercise. However, the load will decrease by $20 \%$ if the pain intensity is higher than $20 \mathrm{~mm} / 100 \mathrm{~mm}$ on the VAS [30]. Only if the patients finish more than $80 \%$ of their target sessions will they be enrolled into the statistical analyses.

Table 1

Treatment protocol performed by the LL group and the BFR groups.

\section{LL group and BFR groups}

Hamstrings stretching, 3 repetitions of $30 \mathrm{~s}$

Bridge with isometric contraction of the transversus abdominis-CORE training, 3 repetitions of $30 \mathrm{~s}^{+}$

Hip abduction with weights (side lying), 3 sets of 10 repetitions $^{+}$

Calm exercises (side lying) with elastic band, 3 sets of 10 repetitions $^{+}$

Calf raises with weights (standing), 3 sets of 10 repetitions $^{+}$

Sensori-motor training (standing) at mini-trampoline, 3 repetitions of $30 \mathrm{~s}$

Leg press (machine), $0^{\circ}-60^{\circ}, 3$ sets of 15 repetitions *

Seated knee extension (machine), $90^{\circ}-0^{\circ}$ of knee flexion, 3 sets of 15 repetitions ${ }^{*}$

* Load is $30 \%$ of the 1-repetition maximum.

+The load will be adjusted every 4 weeks to maintain an effort of perception between 6 and 7 on the Borg scale.

Each group will conduct a warm-up by cycling for 10 minutes before the LLRT or BFR training. The leg press will be performed between $0^{\circ}$ and $60^{\circ}$ of knee flexion. And the leg extension will be performed between $90^{\circ}$ and $45^{\circ}$ of knee flexion [31]. In addition to thigh muscle strength training, distal joint, proximal joint, and core muscle strength training will also be performed for patients with KOA. The reason for this is because the current evidence has shown that biomechanical changes occur in patients with KOA [32] and a combined multi-joint strength exercise is potentially more effective than knee strength training alone in knee musculoskeletal disorders [33]. To avoid possible injuries during the training, we will provide a relative integrated therapeutic exercise program. Thus, stretching exercises and core trainings will also be adopted in this study. During the treatment, we will adopt the 10-point Borg scale to 
monitor the perceived effort for several exercises[34], as shown in the detailed exercise program in Table 1.

The exercise load will be individually set for each participant and be adjusted every 4 weeks by reevaluating the participant's $1 \mathrm{RM}$. Since a direct $1 \mathrm{RM}$ test could potentially cause knee pain or injury in patients with KOA, the 1RM will be estimated by performing a 7-10 RM test, which is the maximum load the individual can complete for 7-10 repetitions. Previous studies have shown that the 7-10 RM test can accurately estimate the 1RM for the leg press [35] and knee extension exercises [36]. The formula is as follows [37]: estimated $1 \mathrm{RM}=$ weight $/(1.0278-0.0278 \times$ reps $)$.

\section{Blood flow restriction exercise}

All participants from the High BFR and Low BFR groups will be individually measured for the LOP and reevaluated every 2 weeks during the study. The LOP will be measured with participants in a relaxed and supine position. The portable color Doppler ultrasound (LOGIQ e, General Electric Company, Boston, USA) will be positioned at the ankle to measure the pedal pulse. A pneumatic cuff $(7-\mathrm{cm}$ width and $56-\mathrm{cm}$ length) will be placed on the proximal thigh of the participant and inflated until the pedal pulse vanishes on the Doppler ultrasound. We will then slowly deflate the cuff, and when the pedal pulse returns, we will record the LOP. The Low BFR group will perform at $40 \%$ LOP, while the High BFR group will perform at $80 \%$ LOP during the exercise. The loads for the leg press and knee extension exercises will be $30 \%$ of the $1 \mathrm{RM}$ (estimated by 7-10 RM). Furthermore, participants in the High BFR and Low BFR groups will perform 1 set of 30 repetitions (or until exhaustion) and 3 sets of 15 receptions with a 30-second interval between sets [20]. The LOP will be adjusted every time to maintain a similar degree of BFR for each individual.

\section{Standardized resistance exercise}

The LL group will conduct a sham BFR in which the pneumatic cuff will be placed on the proximal thigh of the participant with adequate space for two or more fingers between the thigh and cuff. However, there will be no pressure applied in the pneumatic cuff. The exercise protocol of the LL group is the same as the BFR groups. The LL group will perform 3 sets of 15 repetitions with 30\% 1RM and 30-second intervals between sets $[19,21,30]$.

\section{Health education group}

The individuals in the health education group will attend sessions related to protecting their knee joint during daily life. Sessions will meet once a week for 12 weeks. Moreover, we will introduce the basic concept of KOA and the methods used to manage the risks of KOA through various articles from the Internet and leaflets.

\section{Outcome measures}

Primary outcome measures

1) Pain 
The VAS will be used to evaluate pain intensity at rest and under maximum situations during the last week. The VAS is comprised of a line with a length of $100 \mathrm{~mm}$, in which " $0 \mathrm{~mm}$ " corresponds to no pain and "100 mm" to the worst pain imaginable [38]. The VAS will be well depicted for pain, and it is easily operated and widely applied in patients with KOA. The VAS will be used at 0,12 , and 24 weeks after randomization of participants. Furthermore, the minimal clinically important improvement for pain is about $20 \mathrm{~mm} / 100 \mathrm{~mm}$ [39].

2) Self-report function

The knee function self-report outcomes will be measured using the WOMAC. This is a 24-item self-report questionnaire that assesses joint pain, stiffness, and physical function related to KOA [40]. The maximum score of the WOMAC is 120, where a higher score indicates worse symptoms and functions of the knee. The Chinese version of the WOMAC has been shown to be both valid and reliable and also sensitive to changes for patients with KOA [41]. The WOMAC will be measured at 0,12 , and 24 weeks after the randomization of the participants.

\section{3) Adverse events}

All adverse events will be recorded throughout the entirety of the trial. Patients will be made aware of potential adverse events during the consent process and instructed to notify a researcher when adverse events occur. A physiotherapist and related specialists will the categorize adverse events as treatmentrelated or not and the severity of the adverse events within 24 hours. Common BFR or training-related adverse events include muscle soreness, knee pain, a subcutaneous hemorrhage, and numbness.

Secondary outcome measures

1) Quadriceps femoris strength

The quadriceps muscle strength will be evaluated by an isokinetic knee extension with an isokinetic test system (IsoMed 2000, D\&R Ferstl GmbH, Hemau, Germany) device. Before the strength test, the participants will be fastened onto a dynamometric chair in a $90^{\circ}$ sitting position with the torso and thighs fixed by rigid belts. Then, the axis of the dynamometer will be adjusted in alignment with the center of the knee. Furthermore, the range of motion will be set individually for participants by asking them to extend and flex their knee to maximum ranges. Participants will need to perform five constant flexion and extension motions using concentric contractions without a gravity-compensation model at three angular velocities of $60 \%$ second, $90 \%$ second, and $120 \%$ second [42]. During the test, the participants will be encouraged to perform at their maximum effort [43]. The data will be recorded and calculated as the peak torque in Newton-meters, peak torque/body weight, and power in watts. Previous studies have demonstrated that the test-retest reliability for an isometric knee muscle strength assessment is 0.83 in patients with KOA [44].

2) Muscle thickness 
The muscle thickness of the quadriceps will be measured using a portable color Doppler ultrasound [30, 45]. Intra-rater and inter-rater reliabilities have demonstrated good validation when evaluating the muscle by ultrasound. Furthermore, the correlation between an ultrasound and magnetic resonance imaging (MRI) scans for muscle thickness for the vastus medialis, vastus lateralis, and rectus femoris are 0.86 , 0.94 , and 0.86 , respectively $[46,47]$. During the test, the probe will be placed at the mid-belly of these three muscles without depressing the skin. Each muscle will be measured from the adipose tissue-muscle interface to the muscle-bone interface three times. The images will be saved and then averaged. The size of the quadriceps will be estimated as a sum of these three muscles.

3) Physical functional performance

Physical functional performance will be assessed by the timed up and go (TUG) test [48]. Previous studies found that the TUG test has a good intra- and inter-rater reliability ( 0.97 and 0.96 , respectively) for patients with grades 1-3 KOA [49]. During the test, the subject is timed and required to independently rise from an armchair that is $45 \mathrm{~cm}$ in height, walk forward following a straight line for three meters, turn, walk back, and sit down again. The average of three measurements will be used for analysis.

4) Quality of life

The 36-item Short Form Health Survey (SF-36) is a brief self-report questionnaire with 36 questions relevant to QoL in eight health dimensions, including vitality, bodily pain, general health, physical functioning, emotional functioning, social functioning, and mental health in psychometric properties. It can be summarized in two health scores, physical and mental components, where higher scores indicate a better health condition. Previous studies have indicated that the Chinese version of the SF-36 is a relative and valid questionnaire for the general population.

\section{Sample size estimation}

Previous studies have shown the effect size for pain and function are 0.38-0.49 and 0.41-0.52, respectively [50,51]. The sample size was estimated by using G-Power software (version 3.1.9.6, HeinrichHeine-Universität Düsseldorf, Düsseldorf, Germany) with the parameters as follows: analysis of variance (ANOVA) with repeated measures, a type-l error of $5 \%(\alpha=0.05)$, a power of $95 \%(\beta=0.05)$, group numbers $=4$, and the number of measurements $=3$. With a possible dropout rate of $15 \%$, it is estimated that a sample size of 25 patients per group will be needed to verify our study hypotheses for the primary outcomes.

\section{Statistical analyses}

All data will be expressed as mean \pm standard deviation (SD). A linear mixed model with repeated measures will be run to assess the data for fixed factors of the trial (LL group, High BFR group, Low BFR group, and HE group) and time (primary and secondary outcomes). Additionally, two-way ANOVAs will be used to analyze the differences between baseline, post intervention, and follow-up measurements. The analysis will follow an intention-to-treat approach if the subjects drop out during the study. Significance 
will be set at $P<0.05$ for all data. Statistical analyses will be performed using SPSS 22.0 (SPSS Inc., Chicago, USA).

\section{Quality control}

The protocol will be reviewed and revised by experts in orthopedics, physiotherapy, methodology, and statistics. The data will be carefully recorded by both printed and electronic case report forms (eCRFs). Only outcome assessors have access to the eCRFs, and all input data will be double checked by two independent assessors. All data will be unmodifiable once inputted and checked through the eCRF. Only statisticians have access to the database to conduct final statistical analyses. In this trial, both online monitoring and on-site monitoring will be adopted to review the trial processes.

\section{Discussion}

BFR training has become a novel intervention for lower limb degeneration or postsurgical musculoskeletal disorders. However, there have been no consistent recommendations for BFR training in treating KOA in any guidelines until now. In this trial, we will investigate the efficacy of LL RT combined with different BFR pressures $(0 \%, 40 \%$, and $80 \%$ LOP) on pain, self-reported function, physical functional performance, muscle strength, muscle mass, and QoL.

Although several studies have indicated that BFR combined with LLRT is more effective than LLRT alone $[19,20]$, the proper degree of BFR has not been determined. If a 40\% LOP during BFR will produce similar clinical outcomes as an $80 \%$ LOP during BFR in patients with KOA, resistance using a lower LOP can be a choice for those who are intolerant to a high degree of LOP. Additionally, there has been no study comparing the effects of BFR training with a control group without an exercise intervention, and a complete placebo control is not applicable for patients with KOA. Thus, we will set up a control group (the health education group), which will make the study more rigorous and objective. We believe our study will provide the evidence for the effectiveness of RT with BFR, which is important for clinicians in treating patients with KOA.

This trial meets the requirements of methodology for the application of randomization, allocation concealment, an intention-to-treat approach, and masking for patients, assessors, and statisticians. In this trial, there will be 2 sessions per week for 12 weeks, giving a total of 24 exercise sessions for the exercise intervention groups. We will investigate the short-term effects (12 weeks) of these four groups for all clinical outcomes and safety, and the mid-term ( 24 weeks) effects of pain and self-reported function.

We will use the percent of LOP to perform, as similar as possible, the degree of BFR for individuals in each group. Additionally, the LOP will be tested every time before the session to avoid possible changes in hemodynamics of the participants. Considering the potential placebo effect for patients in the LL resistance group, the BFR cuff will also be applied to the participants during the training but the cuff will not be inflated. Moreover, only one study has monitored the pain intensity during training and compared 
the differences between BFR training and HLRT [21]. In this study, we will evaluate the pain intensity during training compared to the tolerance of different degrees of BFR in patients with KOA.

Despite its strengths, this study has some potential limitations. First, due to the nature of the intervention, the physiotherapist cannot be fully masked during the research. Second, like other studies using BFR training, the LOP will be assessed at rest, and it may be altered by changes in body position and muscle contraction. Third, the intervention program consists of core training and stretching in addition to quadriceps training to provide better outcomes for patients with KOA. However, these interventions, especially the stretching exercises, may influence certain outcomes, such as muscle strength. At the end of this research, our results may provide more reliable evidence on the effectiveness of BFR training and determine the proper degree of BFR in treating patients with KOA.

\section{Trial status}

The current approved version of the protocol is version 1.0, March 2021. The recruitment remains in progress. Recruitment will be completed by October 2021 .

\section{Declarations}

\section{Acknowledgements}

We would like to thank Xiaoxiong Zhao from the Beijing Chaoyang Hospital for their help in suggesting the study design and recruitment of participants.

\section{Authors' contributions}

HNW, LC and GXN conceived and designed of the trial. HNW, YC and STW participated in the trial register, communication and monitoring. DXH and LNW participated in the design of statistical analysis. All authors contributed to drafting the manuscript, read and approved the final version.

\section{Funding}

This work was supported by the National Natural Science Foundation of China (81871848).

\section{Availability of data and materials}

The data and the relevant results in this study will be shared through the academic conferences and the scientific papers.

\section{Ethics approval and consent to participate}

The study was approved by the ethics committee of the Beijing Sport University, China. Participants will sign a written informed consent before the intervention. 


\section{Consent for publication}

All authors have agreed to the publication of identifiable data.

\section{Conflict of interest}

All authors declare that they have no conflict of interest relevant to the content of this review.

\section{Abbreviations}

KOA: Knee osteoarthritis; BFR:Blood flow restriction; LLRT:Low-load resistance training; HLRT:HLRT:Lowload resistance training; 1RM:one-repetition maximum; LOP:limb occlusion pressure; SD:Standard deviation; SF-36:Short form 36; VAS:Visual analog scales; TUG:Timed up and go; WOMAC:Western Ontario and McMaster Universities Osteoarthritis Index

\section{References}

1. Sharma L. Osteoarthritis of the Knee. N Engl J Med. 2021;384(1):51-9.

2. Dillon CF, Rasch EK, Gu Q, Hirsch R. Prevalence of knee osteoarthritis in the United States: arthritis data from the Third National Health and Nutrition Examination Survey 1991-94. J Rhuematol. 2006;33(11):2271-9.

3. Tang X, Wang S, Zhan S, Niu J, Tao K, Zhang Y, et al. The Prevalence of Symptomatic Knee Osteoarthritis in China: Results From the China Health and Retirement Longitudinal Study. Arthritis \& rheumatology (Hoboken, NJ). 2016;68(3):648-53.

4. Cross M, Smith E, Hoy D, Nolte S, Ackerman I, Fransen M, et al. The global burden of hip and knee osteoarthritis: estimates from the global burden of disease 2010 study. Ann Rheum Dis. 2014;73(7):1323-30.

5. Neogi T, Zhang Y. Epidemiology of osteoarthritis. Rheum Dis Clin North Am. 2013;39(1):1-19.

6. Vina ER, Kwoh CK. Epidemiology of osteoarthritis: literature update. Curr Opin Rheumatol. 2018;30(2):160-7.

7. Johnson VL, Hunter DJ. The epidemiology of osteoarthritis. Best Pract Res Clin Rheumatol. 2014;28(1):5-15.

8. Takagi S, Omori G, Koga H, Endo K, Koga Y, Nawata A, et al. Quadriceps muscle weakness is related to increased risk of radiographic knee OA but not its progression in both women and men: the Matsudai Knee Osteoarthritis Survey. Knee surgery, sports traumatology, arthroscopy: official journal of the ESSKA. 2018;26(9):2607-14.

9. Dell'isola A, Wirth W, Steultjens M, Eckstein F, Culvenor AG. Knee extensor muscle weakness and radiographic knee osteoarthritis progression. Acta Orthop. 2018;89(4):406-11.

10. Muraki S, Akune T, Teraguchi M, Kagotani R, Asai Y, Yoshida M, et al. Quadriceps muscle strength, radiographic knee osteoarthritis and knee pain: the ROAD study. BMC Musculoskelet Disord. 
2015;16:305.

11. Lee SY, Ro HJ, Chung SG, Kang SH, Seo KM, Kim DK. Low Skeletal Muscle Mass in the Lower Limbs Is Independently Associated to Knee Osteoarthritis. PLoS One. 2016;11(11):e0166385.

12. Cheon YH, Kim HO, Suh YS, Kim MG, Yoo WH, Kim RB, et al. Relationship between decreased lower extremity muscle mass and knee pain severity in both the general population and patients with knee osteoarthritis: Findings from the KNHANES V 1-2. PLoS One. 2017;12(3):e0173036.

13. Liao CD, Chen HC, Kuo YC, Tsauo JY, Huang SW, Liou TH. Effects of Muscle Strength Training on Muscle Mass Gain and Hypertrophy in Older Adults With Osteoarthritis: A Systematic Review and Meta-Analysis. Arthritis Care Res. 2020;72(12):1703-18.

14. American College of Sports Medicine position stand. Progression models in resistance training for healthy adults. Med Sci sports Exerc. 2009;41(3):687-708.

15. Jan MH, Lin JJ, Liau JJ, Lin YF, Lin DH. Investigation of clinical effects of high- and low-resistance training for patients with knee osteoarthritis: a randomized controlled trial. Physical therapy. 2008;88(4):427-36.

16. Takarada Y, Nakamura Y, Aruga S, Onda T, Miyazaki S, Ishii N. Rapid increase in plasma growth hormone after low-intensity resistance exercise with vascular occlusion. J Appl Physiol (1985). 2000;88(1):61-5.

17. Reeves GV, Kraemer RR, Hollander DB, Clavier J, Thomas C, Francois M, et al. Comparison of hormone responses following light resistance exercise with partial vascular occlusion and moderately difficult resistance exercise without occlusion. J Appl Physiol (1985). 2006;101(6):161622.

18. Moore DR, Burgomaster KA, Schofield LM, Gibala MJ, Sale DG, Phillips SM. Neuromuscular adaptations in human muscle following low intensity resistance training with vascular occlusion. Eur J Appl Physiol. 2004;92(4-5):399-406.

19. Segal N, Davis MD, Mikesky AE. Efficacy of Blood Flow-Restricted Low-Load Resistance Training For Quadriceps Strengthening in Men at Risk of Symptomatic Knee Osteoarthritis. Geriatr Orthop Surg Rehabil. 2015;6(3):160-7.

20. Segal NA, Williams GN, Davis MC, Wallace RB, Mikesky AE. Efficacy of blood flow-restricted, low-load resistance training in women with risk factors for symptomatic knee osteoarthritis. Pm $\mathrm{r}$. $2015 ; 7(4): 376-84$.

21. Bryk FF, Dos Reis AC, Fingerhut D, Araujo T, Schutzer M, Cury Rde P, et al. Exercises with partial vascular occlusion in patients with knee osteoarthritis: a randomized clinical trial. Knee surgery, sports traumatology, arthroscopy:. official journal of the ESSKA. 2016;24(5):1580-6.

22. Ferraz RB, Gualano B, Rodrigues R, Kurimori CO, Fuller R, Lima FR, et al. Benefits of Resistance Training with Blood Flow Restriction in Knee Osteoarthritis. Med Sci sports Exerc. 2018;50(5):897905.

23. Jessee MB, Buckner SL, Dankel SJ, Counts BR, Abe T, Loenneke JP. The Influence of Cuff Width, Sex, and Race on Arterial Occlusion: Implications for Blood Flow Restriction Research. Sports Med. 
2016;46(6):913-21.

24. Loenneke JP, Fahs CA, Rossow LM, Sherk VD, Thiebaud RS, Abe T, et al. Effects of cuff width on arterial occlusion: implications for blood flow restricted exercise. Eur J Appl Physiol. 2012;112(8):2903-12.

25. Soligon SD, Lixandrão ME, Biazon T, Angleri V, Roschel H, Libardi CA. Lower occlusion pressure during resistance exercise with blood-flow restriction promotes lower pain and perception of exercise compared to higher occlusion pressure when the total training volume is equalized. Physiol Int. 2018;105(3):276-84.

26. Chan AW, Tetzlaff JM, Altman DG, Laupacis A, Gøtzsche PC, Krleža-Jerić K, et al. SPIRIT 2013 statement: defining standard protocol items for clinical trials. Ann Intern Med. 2013;158(3):200-7.

27. Zwarenstein M, Treweek S, Gagnier JJ, Altman DG, Tunis S, Haynes B, et al. Improving the reporting of pragmatic trials: an extension of the CONSORT statement. BMJ. 2008;337:a2390.

28. Fernandes L, Hagen KB, Bijlsma JW, Andreassen O, Christensen P, Conaghan PG, et al. EULAR recommendations for the non-pharmacological core management of hip and knee osteoarthritis. Ann Rheum Dis. 2013;72(7):1125-35.

29. Kellgren JH, Lawrence JS. Radiological assessment of osteo-arthrosis. Ann Rheum Dis. 1957;16(4):494-502.

30. Giles L, Webster KE, McClelland J, Cook JL. Quadriceps strengthening with and without blood flow restriction in the treatment of patellofemoral pain: a double-blind randomised trial. Br J Sports Med. 2017;51(23):1688-94.

31. Powers CM, Ho KY, Chen YJ, Souza RB, Farrokhi S. Patellofemoral joint stress during weight-bearing and non-weight-bearing quadriceps exercises. J Orthop Sports Phys Ther. 2014;44(5):320-7.

32. Astephen JL, Deluzio KJ, Caldwell GE, Dunbar MJ. Biomechanical changes at the hip, knee, and ankle joints during gait are associated with knee osteoarthritis severity. Journal of orthopaedic research: official publication of the Orthopaedic Research Society. 2008;26(3):332-41.

33. Hislop AC, Collins NJ, Tucker K, Deasy M, Semciw Al. Does adding hip exercises to quadriceps exercises result in superior outcomes in pain, function and quality of life for people with knee osteoarthritis? A systematic review and meta-analysis. Br J Sports Med. 2020;54(5):263-71.

34. Thomas SG, Pagura SM, Kennedy D. Physical activity and its relationship to physical performance in patients with end stage knee osteoarthritis. J Orthop Sports Phys Ther. 2003;33(12):745-54.

35. Reynolds JM, Gordon TJ, Robergs RA. Prediction of one repetition maximum strength from multiple repetition maximum testing and anthropometry. Journal of strength conditioning research. 2006;20(3):584-92.

36. Abadie BR, Wentworth MC. Prediction of one repetition maximal strength from a 5-10 repetition submaximal strength test in college-aged females. Journal of Exercise Physiology Online. 2000;3(3).

37. McNair PJ, Colvin M, Reid D. Predicting maximal strength of quadriceps from submaximal performance in individuals with knee joint osteoarthritis. Arthritis Care Res. 2011;63(2):216-22. 
38. Wewers ME, Lowe NK. A critical review of visual analogue scales in the measurement of clinical phenomena. Res Nurs Health. 1990;13(4):227-36.

39. Tubach F, Ravaud P, Baron G, Falissard B, Logeart I, Bellamy N, et al. Evaluation of clinically relevant changes in patient reported outcomes in knee and hip osteoarthritis: the minimal clinically important improvement. Ann Rheum Dis. 2005;64(1):29-33.

40. Bellamy N, Buchanan WW, Goldsmith CH, Campbell J, Stitt LW. Validation study of WOMAC: a health status instrument for measuring clinically important patient relevant outcomes to antirheumatic drug therapy in patients with osteoarthritis of the hip or knee. J Rhuematol. 1988;15(12):1833-40.

41. Symonds T, Hughes B, Liao S, Ang Q, Bellamy N. Validation of the Chinese Western Ontario and McMaster Universities Osteoarthritis Index in Patients From Mainland China With Osteoarthritis of the Knee. Arthritis Care Res. 2015;67(11):1553-60.

42. Harper SA, Roberts LM, Layne AS, Jaeger BC, Gardner AK, Sibille KT, et al. Blood-Flow Restriction Resistance Exercise for Older Adults with Knee Osteoarthritis: A Pilot Randomized Clinical Trial. Journal of clinical medicine. 2019;8(2).

43. Hall M, Hinman RS, Wrigley TV, Kasza J, Lim BW, Bennell KL. Knee extensor strength gains mediate symptom improvement in knee osteoarthritis: secondary analysis of a randomised controlled trial. Osteoarthritis cartilage. 2018;26(4):495-500.

44. Wessel J. Isometric strength measurements of knee extensors in women with osteoarthritis of the knee. J Rhuematol. 1996;23(2):328-31.

45. Kim D, Loenneke JP, Thiebaud RS, Abe T, Bemben MG. The acute muscular effects of cycling with and without different degrees of blood flow restriction. Acta physiologica Hungarica. 2015;102(4):428-41.

46. Giles LS, Webster KE, McClelland JA, Cook J. Can ultrasound measurements of muscle thickness be used to measure the size of individual quadriceps muscles in people with patellofemoral pain? Physical therapy in sport: official journal of the Association of Chartered Physiotherapists in Sports Medicine. 2015;16(1):45-52.

47. Giles LS, Webster KE, McClelland JA, Cook J. Atrophy of the Quadriceps Is Not Isolated to the Vastus Medialis Oblique in Individuals With Patellofemoral Pain. J Orthop Sports Phys Ther. 2015;45(8):613-9.

48. Podsiadlo D, Richardson S. The timed "Up \& Go": a test of basic functional mobility for frail elderly persons. J Am Geriatr Soc. 1991;39(2):142-8.

49. Alghadir A, Anwer S, Brismée JM. The reliability and minimal detectable change of Timed Up and Go test in individuals with grade 1-3 knee osteoarthritis. BMC Musculoskelet Disord. 2015;16:174.

50. Fransen M, McConnell S, Harmer AR, Van der Esch M, Simic M, Bennell KL. Exercise for osteoarthritis of the knee: a Cochrane systematic review. Br J Sports Med. 2015;49(24):1554-7.

51. Jansen MJ, Viechtbauer W, Lenssen AF, Hendriks EJ, de Bie RA. Strength training alone, exercise therapy alone, and exercise therapy with passive manual mobilisation each reduce pain and 
disability in people with knee osteoarthritis: a systematic review. Journal of physiotherapy. 2011;57(1):11-20.

Figures

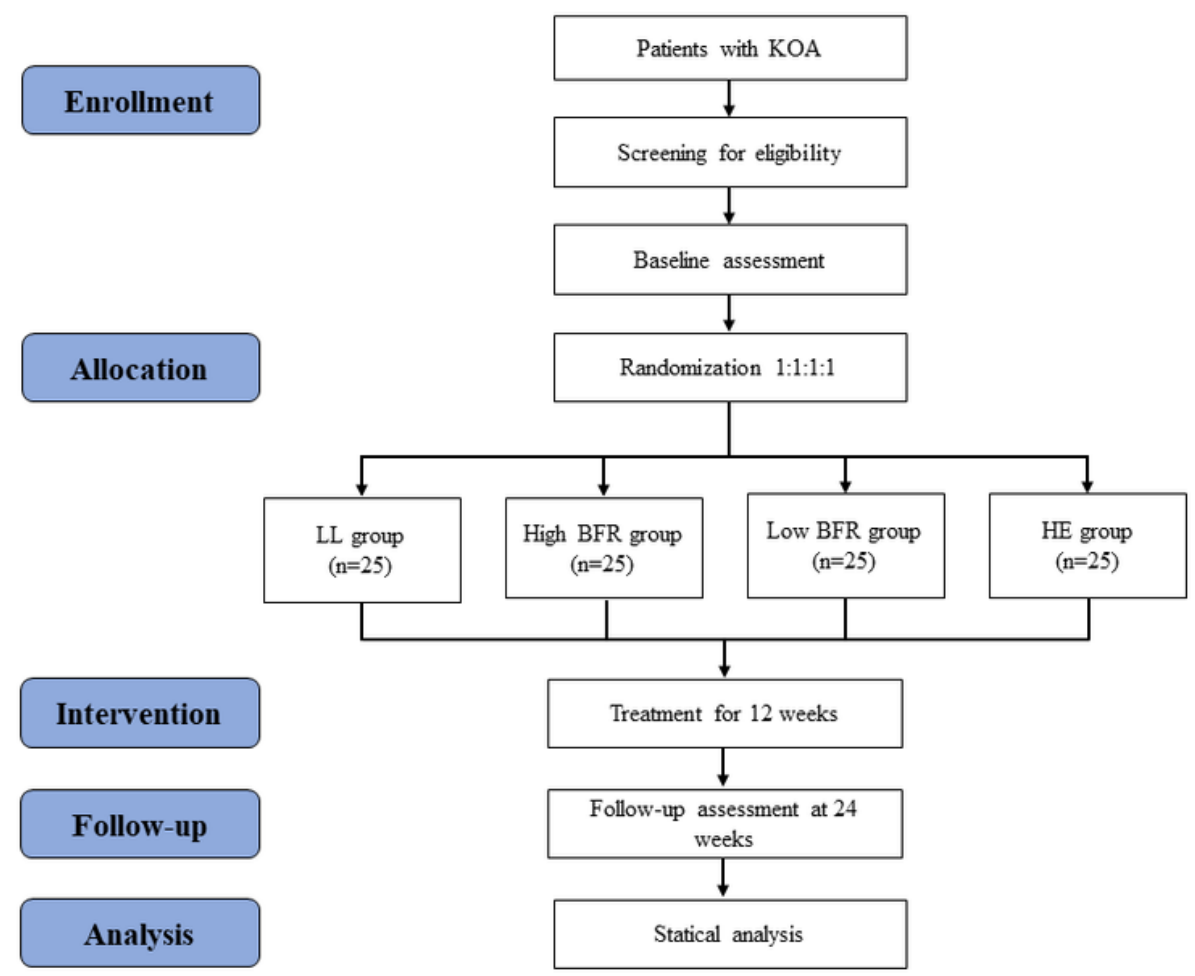

Figure 1

Flow diagram of the planned study. KOA: knee osteoarthritis; LL: low load; BFR: blood flow restriction; HE: health education. 


\begin{tabular}{|c|c|c|c|c|c|c|}
\hline & & \multicolumn{5}{|c|}{ Study period } \\
\hline & & \multirow{2}{*}{$\begin{array}{c}\text { Screening } \\
\text { Week -2 }\end{array}$} & \multirow{2}{*}{$\begin{array}{l}\text { Baseline } \\
\text { Week } 0\end{array}$} & \multirow{2}{*}{$\begin{array}{c}\text { Twelve weeks } \\
\text { Week } 12\end{array}$} & \multicolumn{2}{|c|}{ Follow-up } \\
\hline \multicolumn{2}{|c|}{ Time point } & & & & Week 13 & Week 24 \\
\hline \multirow{3}{*}{ Enrollment } & Eligibility screen & $x$ & & & & \\
\hline & Informed consent & $x$ & & & & \\
\hline & Allocation & & $x$ & & & \\
\hline \multirow{4}{*}{ Intervention } & LL group & & & $\longrightarrow$ & & \\
\hline & High BFR group & & & $\longleftrightarrow$ & & \\
\hline & Low BFR group & & & $\longleftrightarrow$ & & \\
\hline & Health education group & & & $\longrightarrow$ & & \\
\hline \multirow{7}{*}{ Assessment } & Pain & & $x$ & & $x$ & $x$ \\
\hline & Self-reported function & & $x$ & & $x$ & $x$ \\
\hline & Physical function performance & & $x$ & & $\times$ & \\
\hline & Muscle strength & & $x$ & & $x$ & \\
\hline & Muscle mass & & $\times$ & & $\times$ & \\
\hline & Quality of life & & $x$ & & $x$ & \\
\hline & Adverse events & & $x$ & & $x$ & \\
\hline
\end{tabular}

\section{Figure 2}

Schedule of enrollment, intervention, and assessment (SPIRIT Figure). LL, low load; BFR, blood flow restriction.

\section{Supplementary Files}

This is a list of supplementary files associated with this preprint. Click to download.

- Additionalfile1SPIRITchecklist.docx 